\title{
Filhos das fronteiras: revisão de literatura sobre imigração involuntária, infância e saúde mental
}

\section{Children of the Borders: Literary Review Regarding Involuntary Immigration, Childhood and Mental Health}

Hijos de las fronteras: revisión de literatura sobre inmigración involuntaria, infancia y salud mental

\author{
Cecília Braga Bezerra ${ }^{1}$ Lattes, Lucienne Martins Borges ${ }^{2}$ Lattes, Maiara Pereira Cunha ${ }^{3}$ Lattes \\ 12 Universidade Federal de Santa Catarina, \\ ${ }^{3}$ Universidade do Vale do Itajaí \\ Brasil
}

Fecha correspondencia:

Recibido: marzo 5 de 2018.

Aceptado: octubre 22 de 2018.

Forma de citar:

Bezerra, C. B., Martins-Borges, L.,

\& Cunha, M. P. (2019). Filhos das

fronteiras: revisão de literatura

sobre imigração involuntária,

infância e saúde mental. Rev.CES

Psico, 12(2), 26-40.

Open access

(c) Copyright

Licencia creative commons

Etica de publicaciones

Revisión por pares

Gestión por Open Journal System

DOl: http://dx.doi.org/10.21615/

cesp.12.2.3

ISSN: 2011-3080
Comparte

\section{Resumo}

$\mathrm{Na}$ atualidade há um expressivo contingente de refugiados que deixam suas casas devido a conflitos -guerras, perseguições e violações dos direitos humanos- em busca de novas oportunidades. O Brasil tem se tornado um dos destinos destas pessoas, inclusive recebendo crianças refugiadas. Este estudo objetiva revisar a literatura científica disponível em bases de dados eletrônicas nos últimos treze anos a respeito dos impactos psicológicos da imigração involuntária em crianças. A pesquisa foi realizada em cinco bases de dados (BVS-PSI, Periódicos Capes, Francis, Redalyc e PsycARTICLES), a partir da interseção dos descritores "immigration", "children" e "mental health". Selecionou-se 48 artigos para análise, sendo constatada a ausência de publicações nacionais. Os conteúdos abordados nos trabalhos foram agrupados constituindo três categorias, a saber: 1) desafios enfrentados pelas crianças ao chegar ao país de acolhimento, 2) impactos psicológicos do processo migratório forçado na infância e 3) propostas de intervenção na saúde mental dos pequenos refugiados. A partir das informações obtidas, enfatiza-se a elaboração de políticas públicas no contexto brasileiro, sobretudo direcionadas a Atenção Básica com vistas a considerar as singularidades da criança refugiada no seu acolhimento.

Palavras-chave: Refugiados, Imigrantes, "Imigração Involuntária", Deslocadas, Infância, Crianças, Saúde Mental.

\section{Abstract}

Nowadays, a significant number of refugees who depart from their homes due to conflicts such as war, persecution, and human rights violations are searching for new opportunities. Brazil has become one of these receiving countries that not only is assisting adult refugees but also children. This study aims to review the scientific literature available on electronic data- 


\section{Sobre los autores:}

1. Mestre em Psicologia. Núcleo de Estudos sobre Psicologia, Migrações e Culturas, Centro de Filosofia e Ciências Humanas Universidade Federal de Santa Catarina.

2. Doutora em Psicologia. Professora titular do Departamento de Psicologia da Universidade Federal de Santa Catarina - UFSC, coordenadora do Núcleo de Estudos sobre Psicologia, Migrações e Culturas NEMPsiC,

3. Doutora em Psicologia. Professora da Universidade do Vale do Itajaí. bases in the last thirteen years about the psychological impact of involuntary migration in children. The research was conducted in five databases (BVS-PSI, Capes Journals, Francis, Redalyc and PsycARTICLES), from the intersection of "immigration", "children" and "mental health" descriptors. For the analysis, forty-five articles were selected, and it was noticed the lack of national publications (Brazil). The contents addressed in the works were grouped into three categories: 1) challenges faced by children during their arrival in the receiving country, 2) psychological impacts of forced migration process in childhood, 3) intervention proposals on mental health of refugee children. Based on the information obtained, it was stressed the formulation of public policies in the Brazilian context, focused on Basic Health Care and particular needs of the refugee children by the time of their reception.

Keywords: Refugees, Immigrants, Involuntary Immigration, Displaced, Childhood, Children, Mental Health.

\section{Resumen}

En la actualidad, existe una cantidad significativa de refugiados que abandonan sus hogares debido a conflictos: -guerra, persecución y violaciones de los derechos humanos- en busca de nuevas oportunidades. Brasil se ha convertido en uno de los destinos de estas personas, incluso recibiendo niños refugiados. Este estudio tiene como objetivo revisar la literatura científica disponible en bases de datos electrónicas en los últimos trece años sobre el impacto psicológico de la migración involuntaria en niños. La investigación se realizó en cinco bases de datos (BVS-PSI, Capes Journals, Francis, Redalyc y PsycARTICLES), a partir de la intersección de los descriptores "inmigración", "niños" y "salud mental". Se seleccionaron 45 artículos para su análisis, siendo constatada la ausencia de publicaciones nacionales (Brasil). Los contenidos abordados en los trabajos se agruparon en tres categorías: 1) desafíos que enfrentan los niños al llegar al país de acogida, 2) impactos psicológicos del proceso de migración forzosa en la infancia y 3 ) propuestas de intervención en la salud mental de los niños refugiados. Con base en la información obtenida, se enfatiza la elaboración de políticas públicas en el contexto brasileño, principalmente dirigidas a Atención Básica con vistas a considerar las singularidades del niño refugiado en su proceso de acogida.

Palabras clave: Refugiados, Inmigrantes, Inmigración Involuntaria, Desplazados, Infancia, Niños, Salud Mental.

\section{Introdução}

Pequenos bebês envoltos em capas não surgem apenas nos desenhos animados como super-heróis, mas se multiplicam nos noticiários internacionais sendo cobertos por invólucros da guarda-costeira após, heroicamente, sobreviverem às travessias do Mediterrâneo em botes clandestinos. Com o número crescente de deslocamento global provocado por guerras, conflitos, perseguições -65.6 milhões de pessoas foram forçadas a deixar suas casas até o final de 2017 -, sendo que $51 \%$ delas possuem menos de 18 anos de idade, assim sendo, a temática da imigração se popularizou, os refugiados se tornaram pauta desde os fóruns internacionais e debates acadêmicos às conversas cotidianas do grupo de amigos (Comitê Nacional para os Refugiados (CONARE), 2017; United Nations High Commissioner for Refugees, 2017).

No território brasileiro os índices também aumentam, com crescimento nas solicitações de refúgio de mais de $930 \%$ entre 2010 e 2013. Ao final do ano de 2016, contabilizou-se 10.308 solicitações de refúgios, dentre os quais 9.552 foram devidamente 
Pág 28

Os impactos psicológicos das experiências traumáticas associadas à imigração involuntária na infância eclodem em pesadelos, isolamento social, baixo rendimento escolar e sentimento de tristeza, assim como, alterações emocionais e comportamentais interferem no desenvolvimento infantil e denotam o sofrimento psicológico que acomete crianças em refúgio (Erol, Simsek, Öner, \& Munir, 2005; Flink et al., 2013; Kronick \& Rousseau, 2015; Lien, Haavet, Thoresen, Heyerdahl, \& Bjertness, 2007; Lipsicas \& Mäkinen, 2010; Nielsen et al., 2008). reconhecidas. Por sua vez, os pequenos imigrantes -crianças de 0 a 12 anos de idade- representam 7\% dos refúgios deferidos (CONARE, 2017). Na companhia de seus pais em busca da sobrevivência ou vivendo a perda de estar desacompanhada, as crianças procuram guarida no país de acolhimento, marcadas por feridas que vão além das cicatrizes corporais (Bezerra, 2016).

Os impactos psicológicos das experiências traumáticas associadas à imigração involuntária na infância eclodem em pesadelos, isolamento social, baixo rendimento escolar e sentimento de tristeza, assim como, alterações emocionais e comportamentais interferem no desenvolvimento infantil e denotam o sofrimento psicológico que acomete crianças em refúgio (Erol, Simsek, Öner, \& Munir, 2005; Flink et al., 2013; Kronick \& Rousseau, 2015; Lien, Haavet, Thoresen, Heyerdahl, \& Bjertness, 2007; Lipsicas \& Mäkinen, 2010; Nielsen et al., 2008). Somam-se, ainda, as dificuldades enfrentadas diante do convívio simultâneo com a cultura de onde vieram e aquela do país anfitrião, cujo encontro de diferenças potencializa os riscos de problemas de saúde mental e tornam crianças e jovens imigrantes mais vulneráveis psicologicamente (Antunes, 2017; Becker \& Martins-Borges, 2015; Martins-Borges, 2017; Moleiro \& Goncalves, 2010; Pumariega, Rogers, \& Rothe, 2005; Skokauskas \& Clarke, 2009).

Diante do contexto aqui exposto, parece urgente atentar para as questões da saúde mental dos pequenos. Desse modo, o presente artigo se constitui com o propósito de apresentar uma revisão da literatura acerca dos impactos psicológicos da imigração involuntária na infância. Faz-se mister evidenciar o panorama das publicações científicas sobre as repercussões psicológicas da imigração na infância visando fomentar a discussão com o meio acadêmico e a comunidade.

\section{Método}

Realizou-se uma revisão de literatura com o propósito de conhecer o panorama das publicações científicas acerca dos impactos psicológicos da imigração involuntária em crianças. A partir dessa questão, iniciou-se a etapa de localização e seleção dos estudos que poderiam elucidá-la. Optou-se pela busca em cinco bases de dados eletrônicas: (1) Biblioteca Virtual em Saúde e Psicologia [BVS-PSI]; (2) Portal de Periódicos Capes; (3) Francis; (4) Rede de Revistas Científicas da América Latina, do Caribe, da Espanha e de Portugal [Redalyc]; e plataforma da (5) American Psychological Association [APA PsycARTICLES]. As bases de dados foram escolhidas com intuito de abarcar diferentes regiões de produção científica no mundo e por se tratarem de referências quanto às publicações virtuais, sendo que a pesquisa se deu nas cinco plataformas separadamente.

Posteriormente, consultou-se a Terminologia em Psicologia da BVS-Psi e os Descritores em Ciências da Saúde (DeCS) da Biblioteca Virtual em Sáude (BVS) para definir quais descritores melhor se adequariam ao estudo. Rastreios prévios foram feitos nas referidas bases a partir de terminologias escritas em português, contudo, a amostra localizada se mostrou escassa e com pouca aproximação à temática de pesquisa. Escolheu-se, então, o uso no idioma inglês dos descritores "immigration", "children" e "mental health", que foram inseridos nessa ordem e conectados pelo operador booleano "AND", de forma a recuperar a interseção dos termos e garantir que todos os conceitos estivessem presentes.

A busca nas bases de dados a partir dos descritores foi feita em janeiro de 2018, acrescentando-se como filtro à pesquisa que os estudos fossem referentes ao período 
de janeiro de 2005 até a data em questão com intuito de obter o maior número de informações. Além disso, indicou-se como critério que as produções deveriam apenas se tratar de artigos científicos. Não houve restrição quanto ao idioma de publicação dos artigos, mas se percebeu que os trabalhos foram escritos em inglês, francês e espanhol. Localizou-se, inicialmente, o total de 157 publicações, cujos resumos foram lidos com a finalidade de excluir os estudos em repetição ou aqueles que não se tratavam de artigos científicos, e verificar se os mesmos estavam diretamente relacionados às temáticas da imigração involuntária, infância e saúde mental.

Observou-se que boa parte dos artigos excluídos por não se relacionarem à temática $(N=35)$ versava sobre pesquisas em que o foco estava nos pais de famílias imigrantes e outra quantidade importante $(\mathrm{N}=20)$ abordava os efeitos da imigração em participantes adultos. Houve ainda estudos que discutiam aspectos migratórios gerais ( $N=13)$, sem especificação relacionada à imigração na infância; e artigos cuja temática da imigração tinha papel secundário $(N=12)$, em investigações voltadas para maus-tratos à criança, capacitação de profissionais de saúde, estratégias de urbanização de favelas e saúde reprodutiva de mulheres.

Após essa triagem, percebeu-se a necessidade de excluir também artigos publicados fora do período determinado - apesar da discriminação no filtro de busca. Ao fim, foram selecionados 48 artigos, cujo fluxo do processo de seleção é ilustrado na Figura 1.

Figura 1. Processo de localização e seleção de artigos

\begin{tabular}{|c|c|}
\hline \multicolumn{2}{|c|}{$\begin{array}{c}\text { Seleção das bases de dados } \\
\text { BVS-PSI, Periódicos Capes, Francis, Redalyc, PsycNET }\end{array}$} \\
\hline \multicolumn{2}{|c|}{$\begin{array}{l}\text { Busca a partir dos descritores } \\
\text { "immigration", "children" e "mental health" }\end{array}$} \\
\hline & \begin{tabular}{|c|} 
Artigos localizados \\
Total: 157 \\
\end{tabular} \\
\hline \multicolumn{2}{|c|}{$\begin{array}{l}\text { Critérios de inclusão } \\
\text { Publicado na última década, ser artigo científico, ter relação direta com temática }\end{array}$} \\
\hline & $\begin{array}{l}\quad \text { Artigos excluídos } \\
\text { Repetidos: } 07 \\
\text { Não são artigos: } 15 \\
\text { Sem relação temática: } 80 \\
\text { Data distinta do período: } 07\end{array}$ \\
\hline $\begin{array}{r}\text { Artigos sel } \\
\text { Total } \\
\end{array}$ & $\begin{array}{l}\text { ecionados } \\
48\end{array}$ \\
\hline
\end{tabular}

Partiu-se para a leitura integral dos artigos selecionados, com elaboração de fichas sintéticas sobre o conteúdo das produções, de modo a permitir a identificação das seguintes variáveis para análise: data de publicação, local onde o estudo foi realizado, população estudada, natureza da pesquisa -e teórica ou empírica-, abordagem da pesquisa, técnica utilizada de coleta de dados - para os estudos empíricos-, e temas centrais abordados. A fase de análise dos dados seguiu com o agrupamento de artigos em eixos temáticos de acordo com as similaridades dos temas centrais por ele abordados na tentativa de organizar os dados para apresentação dos principais resultados. Os eixos temáticos criados foram: 1) Desafios no país de acolhimento, neste item concentram-se estudos relacionados a aspectos sociais, econômicos, educacionais e transgeracionais que interferem no processo de adaptação das crianças; 2) Im- 
Pág 30

O número de produções científicas a partir do objetivo deste estudo demonstrou não ter acompanhado a vertiginosa população, haja vista que no ano de 2016 foram encontradas duas publicações e, no ano de 2017 apenas uma. pactos Psicológicos, neste item os artigos enfocam a investigação, caracterização e discussão acerca das repercussões psicológicas advindas do processo migratório; e 3) Propostas de intervenção, neste item foram agrupados os trabalhos voltados para a validação de instrumentos para mensurar as repercussões psicológicas e para a proposição ou relato de experiências acerca de intervenções realizadas com crianças imigrantes.

Diante do arcabouço de dados coletados a partir da leitura analítica dos artigos, fez-se a interpretação dos resultados, analisando-os articuladamente com base no objetivo de pesquisa. As considerações finais foram elaboradas com indicações de possíveis desdobramentos que o panorama das publicações científicas identificado pode refletir no estudo acerca dos impactos psicológicos da imigração involuntária em crianças.

\section{Resultados e discussão}

Os artigos selecionados nesta pesquisa foram produzidos em países de diferentes continentes, com produções oriundas dos Estados Unidos ( $N=19)$, do Canadá ( $N=09$ ), da Inglaterra ( $\mathrm{N}=04)$, da Espanha $(\mathrm{N}=02)$, da Holanda $(\mathrm{N}=02)$, da Irlanda $(\mathrm{N}=02)$, da Noruega ( $N=02)$, da Alemanha $(N=02)$, da Argentina $(N=01)$, da Austrália $(N=01)$, da Dinamarca $(N=01)$, do México $(N=01)$, da Suécia $(N=01)$ e da Turquia $(N=01)$. Nota-se a exclusividade de publicações internacionais, sugestiva demanda para o surgimento de contribuições brasileiras sobre a temática, haja vista o crescente índice de imigrantes involuntários no País. A população pesquisada pelos estudos também se mostrou diversificada, havendo estudos que definiram seus grupos populacionais de trabalho nomeando-os como hispânicos $(\mathrm{N}=04)$, latinos $(\mathrm{N}=02)$, mexicanos $(\mathrm{N}=02)$, afegãos $(N=01)$, asiáticos $(N=01)$, colombianos $(N=01)$, de minoria racial $(N=01)$, filipinos ( $N=01)$, não-ocidentais $(N=01)$, somalis $(N=02)$, turcos $(N=02)$ e vietnamitas $(\mathrm{N}=01)$. Faz-se destaque para os artigos que trabalharam com populações de múltiplas nacionalidades $(\mathrm{N}=16)$, que incluem sírios, caribenhos, chineses, dominicanos, russos; e aqueles em que não houve especificação da nacionalidade dos imigrantes em questão $(\mathrm{N}=16)$.

No que se refere à data de publicação desses artigos, percebe-se, uma maior concentração nos anos de 2012 e 2014, com a publicação de 07 artigos em cada, seguidos do ano de 2007, quando 06 publicações foram lançadas. Ressalta-se que, dentre os artigos selecionados para esta revisão, não houve publicação no período de 2011 . No ano de 2014, houve um marco histórico de pessoas em deslocamento ao registrar-se 59 milhões de refugiados, requerentes de asilo e deslocados internos no mundo, índice que não era alcançado desde a Segunda Guerra Mundial (Alto Comissariado das Nações Unidas para Refugiados, 2014). No entanto, o número de produções científicas a partir do objetivo deste estudo demonstrou não ter acompanhado a vertiginosa população, haja vista que no ano de 2016 foram encontradas duas publicações e, no ano de 2017 apenas uma.

A presente revisão procurou identificar, também, qual a natureza das pesquisas sobre saúde mental de crianças imigrantes. Observou-se a que a maioria dos trabalhos ( $N=35)$ utilizou métodos de coleta de dados com pessoas como participantes, caracterizando-se como pesquisas empíricas. Os demais 13 estudos foram constituídos de revisões de literatura e discussões teóricas relacionadas à temática da saúde mental na imigração infantil. Esses últimos estudos não foram classificados quanto à abordagem da pesquisa e técnicas utilizadas na coleta de dados. 
Dentre os 35 estudos de natureza empírica, observou-se que a maior parte deles fez uso de abordagem quantitativa $(\mathrm{N}=25)$, em detrimento de $(\mathrm{N}=8)$ estudos de cunho qualitativo, e daqueles de abordagem mista, ou seja, ( $N=2)$ pesquisas de abordagem quanti-quali. Cabe refletir se a escolha pelo delineamento quantitativo pode revelar melhor adequação aos objetivos estudados ou se trata de uma tradição específica de predileção pela abordagem em cada país, a exemplo dos Estados Unidos, que produziram o maior número de artigos aqui analisados, cujo histórico de pesquisas em psicologia é marcado pela abordagem quantitativa (Perdigão, Herlinger, \& White, 2012).

Quanto às técnicas utilizadas na coleta de dados de tais pesquisas, pode-se observar na Tabela 1 o predomínio de questionários como recurso principal dos estudos empíricos. Contudo, ressalta-se que a diversidade de técnicas parece refletir a necessidade de diferentes métodos para alcançar as populações de culturas distintas.

Tabela 1. Distribuição dos artigos empíricos quanto às técnicas utilizadas na coleta de dados

\begin{tabular}{ll}
\hline \multicolumn{1}{c}{ Técnica utilizada na coleta de dados } & N \\
\hline Questionário & 23 \\
Questionário e auto-relato & 01 \\
Entrevista & 01 \\
Entrevista e questionário & 03 \\
Entrevista e grupo focal & 02 \\
Entrevista, grupo de discussão e relato comunicativo & 01 \\
Entrevista e observação etnográfica & 01 \\
Grupo focal & 01 \\
Estudo de caso & 01 \\
Relato de experiência & 01 \\
Total & $\mathbf{3 5}$ \\
\hline
\end{tabular}

Legenda: Tabela elaborada pelas autoras deste artigo

As múltiplas temáticas presentes nos artigos também anunciam a gama de conteúdos a serem observados quando se trata de questões psicológicas na imigração infantil. Com o propósito de apresentar os principais resultados dos artigos de forma articulada, serão descritos a seguir os eixos temáticos criados para análise dos estudos. A distribuição dos referidos artigos nos eixos de acordo com o tema central abordada no estudo pode ser observada na Tabela 2 através do nome de seus autores.

Tabela 2. Agrupamento dos artigos quanto aos eixos temáticos

\begin{tabular}{|c|c|c|c|}
\hline Eixo temático & Autores & Tema central & $N$ \\
\hline $\begin{array}{l}\text { Desafios no país } \\
\text { de acolhimento }\end{array}$ & $\begin{array}{l}\text { Buriel (2012); Chuang e Gielen (2009); Cook, } \\
\text { Brown, Loder e Wissow (2014); Ellis et al. (2008); } \\
\text { Frankenberg, Kupper, Wagner e Bongard (2013); } \\
\text { Georgiades, Boyle e Duku (2007); González, } \\
\underline{\text { Cuxart e Peco (2012); Hadfield, Ostrowski e }} \\
\underline{\text { Ungar (2017); Lauritzen e Sivertsen (2012); }} \\
\underline{\text { Mace, Mulheron, Jones e Cherian (2014); Pfeifer }} \\
\underline{\text { et al. (2007); Pumariega e Rothe (2010); Spiegler }} \\
\underline{\text { Verkuyten e Leyendecker (2016); Trickett e }} \\
\underline{\text { Jones (2007); Ziol-Guest e Kalil (2012) }}\end{array}$ & $\begin{array}{l}\text { Relação pais-filhos; serviços de saúde; } \\
\text { aculturação; escolarização; contexto ambiental; } \\
\text { convívio escolar; estabelecimento de amizades; } \\
\text { condições ambientais de centros de asilo; } \\
\text { desempenho escolar; identidade social; estresse } \\
\text { de aculturação; mediação cultural; acesso à } \\
\text { saúde }\end{array}$ & 15 \\
\hline
\end{tabular}




\begin{tabular}{|c|c|c|c|}
\hline Eixo temático & Autores & Tema central & $N$ \\
\hline $\begin{array}{l}\text { Impactos } \\
\text { Psicológicos }\end{array}$ & 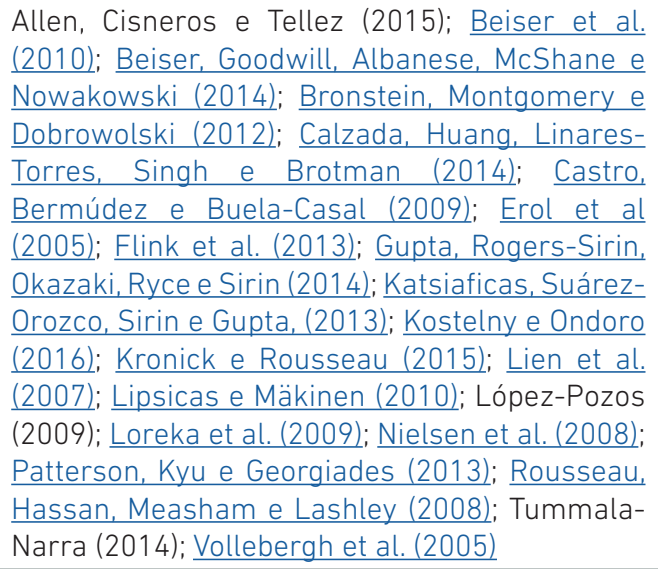 & $\begin{array}{l}\text { Fatores de interferência; pais deportados; } \\
\text { agressão física; TEPT; familismo; condutas } \\
\text { de risco para HIV; deslocamento interno; } \\
\text { deslocamento interno; autoestima coletiva; } \\
\text { estresse de aculturação, depressão e ansiedade; } \\
\text { estresse, angústia e trauma em crianças } \\
\text { detidas; doenças infeciosas agudas; suicídio; } \\
\text { separação e reunificação; crianças detentas; } \\
\text { tempo de espera e mudanças de solicitantes de } \\
\text { asilo; alteração no humor, ansiedade e uso de } \\
\text { substâncias; transtorno de conduta e separação } \\
\text { pais-filhos; identidade étnica; questões } \\
\text { emocionais e comportamentais }\end{array}$ & 21 \\
\hline $\begin{array}{l}\text { Propostas de } \\
\text { intervenção }\end{array}$ &  & $\begin{array}{l}\text { Programas de prevenção; competência cultural; } \\
\text { atenção sensível à cultura; escala para estresse } \\
\text { aculturativo; depressão e estresse cultural; } \\
\text { escala cultural para estresse; escala para } \\
\text { avaliação em consulta pediátrica; pesquisas } \\
\text { e políticas de acolhimento; papel do pediatra; } \\
\text { aspectos culturais em serviços de psiquiatria; } \\
\text { competência cultural; formação de recursos } \\
\text { humanos }\end{array}$ & 12 \\
\hline
\end{tabular}

Legenda: Tabela elaborada pelas autoras deste artigo.

\section{Desafios no país de acolhimento}

Fizeram parte desse eixo 15 artigos, que abordam questões relacionadas ao processo de adaptação das crianças imigrantes ao chegarem ao país que as recebe como refugiadas. Pumariega e Rothe (2010) pontuam que aspectos como aprender a nova língua, renegociar a identidade cultural, e lidar com o isolamento social, o preconceito e a discriminação são desafios enfrentados por pequenos imigrantes. As condições ambientais, o tempo de permanência em campos e abrigos, a escolaridade, e a situação financeira precária também são adversidades percebidas como fatores de risco para saúde mental de crianças solicitantes de asilo (Hadfield, Ostrowski, \& Ungar, 2017; Lauritzen \& Sivertsen, 2012).

Mace, Mulheron, Jones e Cherian (2014), em pesquisa realizada a partir dos registros de consultas pediátricas de serviço de saúde australiano para refugiados, investigaram o histórico educacional das crianças e perceberam que a maior parte delas havia sofrido interrupção da escolaridade e relatava o desenvolvimento da segunda língua como preocupação escolar. 0 incentivo à reinserção escolar e as práticas inclusivas das crianças imigrantes é defendida por González, Cuxart e Peco (2012), Spiegler, Verkuyten, Thijs e Leyendecker (2016) os quais enfatizam a possibilidade de coexistência e o estabelecimento de vínculos entre estudantes nativos e de diferentes origens.

Buriel (2012) aborda o papel especial que as crianças mexicanas desempenham no processo de adaptação das famílias recém-chegadas ao território norte-americano, funcionando como facilitadoras em relação ao idioma e à cultura. Há um debate sobre a mediação cultural produzir inversão de papéis na família e adultificação do adolescente, ou, simplesmente, ser uma das maneiras de crianças e jovens contribuírem 
Pág 33

Frutos do deslocamento, as crianças imigrantes têm sua mente posta em movimento. Sentimentos, ideias, comportamentos que tem o curso modificado de forma tão abrupta quanto o abalo inesperado do terremoto. Experiências traumáticas que irrompem o psiquismo, o luto das perdas que deprime e angustia, as marcas da violência silenciada que fazem o corpo falar na dor. para o funcionamento familiar. Em um estudo com adolescentes vietnamitas, os resultados alcançados por Trickett e Jones (2007) apontam para um cenário misto nessa discussão, ao evidenciar que a mediação cultural não se relacionou à satisfação da família e à coesão familiar; e que houve mais conflito familiar em adolescentes mediadores, mas também uma maior adaptabilidade da família.

Os aspectos da aculturação e o estresse relacionado à adaptação familiar, combinados com o pouco preparo nos serviços para acolher tal grupo pode contribuir para um maior risco de transtornos de saúde mental em crianças e jovens imigrantes tais como: Transtorno de Estresse Pós-Traumático, Transtornos Depressivos e Ansiosos (Frankenberg, Kupper, Wagner, \& Bongard, 2013; Chuang \& Gielen 2009). Em um estudo que investigou as comunicações estabelecidas entre os pais de crianças latinas e os profissionais médicos sobre aspectos psicossociais de seus filhos, Cook, Brown, Loder e Wissow (2014) verificaram que a aculturação tem papel influenciador no estabelecimento de uma boa comunicação, só havendo evolução na confiança para partilhar aspectos da saúde mental das crianças com o passar dos anos.

Pfeifer et al. (2007) atentaram-se para os fatores estressores na chegada ao país de acolhimento, bem como, estudaram as especificidades da construção da identidade social grupal em minorias étnicas, a partir de crianças imigrantes recém-chegadas da China, da União Soviética e da República Dominicana e que passaram a residir no território americano. Para além das questões aculturativas, Ellis, MacDonald, Lincoln e Cabral (2008) sugerem que a discriminação estaria fortemente relacionada a efeitos na saúde mental. Os autores enfatizam que a inserção social é prioritária, e a ausência de vínculos com a comunidade pode afetar o processo adaptativo. Georgiades, Boyle e Duku (2007), ao realizar uma pesquisa no Canadá, constataram o fator resiliência em famílias de imigrantes recentes, que residem em bairros com presença significativa de outros imigrantes, quando o contexto ambiental tende a influenciar positivamente o desempenho escolar e aspectos emocionais e comportamentais das crianças.

\section{Impactos Psicológicos}

Frutos do deslocamento, as crianças imigrantes têm sua mente posta em movimento. Sentimentos, ideias, comportamentos que tem o curso modificado de forma tão abrupta quanto o abalo inesperado do terremoto. Experiências traumáticas que irrompem o psiquismo, o luto das perdas que deprime e angustia, as marcas da violência silenciada que fazem o corpo falar na dor. Os impactos psicológicos da imigração involuntária na criança foram abordados por 21 dos artigos pesquisados, havendo objetivos de estudo focados na identificação de sintomas psíquicos, mas também no comparativo com populações não-imigrantes, e na discussão acerca de tais repercussões psicológicas no desenvolvimento infantil.

Bronstein, Montgomery e Dobrowolski (2012) realizaram um estudo no Reino Unido com crianças afegãs solicitantes de refúgio e verificaram que um terço dos participantes apresentou indicativos de ser suscetível a ter Transtorno de Estresse Pós-traumático (TEPT). Há importantes conexões do TEPT aos eventos traumáticos pré-migratórios que as crianças vivenciaram, associados ao próprio processo da imigração, ao status migratório e às condições de vida e assistência social.

Loreka et al. (2009) avaliaram a saúde mental e física de crianças de um centro de detenção britânico, por meio de entrevistas clínicas estruturadas e questionários de auto-relato, e observaram que todas as crianças participantes relataram sintomas 
Pág 34

A experiência das crianças de famílias detidas no processo de imigração também é foco do estudo de Kronick e Rousseau (2015), que realizaram observação etnográfica participante e entrevistas semi-estruturadas em um centro canadense. A pesquisa apresenta 0 cotidiano das famílias detidas, indica os sentimentos de criminalização e impotência experienciado pelas crianças, além das respostas estressantes e traumáticas à separação de seus pais. de depressão e ansiedade. Problemas no sono, queixas somáticas, falta de apetite, sintomas emocionais e dificuldades comportamentais foram achados comuns. As avaliações pediátricas indicaram que $40 \%$ das crianças apresentaram perda de peso, além de ter havido ocorrências de internamento e faltas a consultas de saúde, o que levantou preocupações quanto ao desenvolvimento, à nutrição e à proteção infantil. A experiência das crianças de famílias detidas no processo de imigração também é foco do estudo de Kronick e Rousseau (2015), que realizaram observação etnográfica participante e entrevistas semi-estruturadas em um centro canadense. A pesquisa apresenta o cotidiano das famílias detidas, indica os sentimentos de criminalização e impotência experienciado pelas crianças, além das respostas estressantes e traumáticas à separação de seus pais.

Ao estudar os efeitos do deslocamento interno e reassentamento no desenvolvimento emocional e comportamental de crianças turcas, Erol et al. (2005) atentaram para variáveis como a idade da criança, o sexo, existência de doença física, situação laboral dos pais e residência da família em áreas urbanas. Os autores observaram que tais fatores foram preditores importantes para a internalização e externalização de problemas, havendo relação entre o deslocamento e a maior experimentação dos problemas. Com crianças deslocadas internamente na Somália, Kostelny e Ondoro (2016) destacam que para além da guerra, as crianças cotidianamente enfrentavam situações estressantes, dentre quais: negligência, trabalho infantil, evasão escolar e/ou uso de substâncias psicoativas vivenciando um acúmulo de situações conflitantes. Também foi possível perceber pelo estudo de Flink et al. (2013) que a saúde mental das crianças deslocadas internas na Colômbia se mostrou pior que a de crianças não-deslocadas.

Em contraponto ao cenário majoritário dos trabalhos revisados, Vollebergh et al. (2005) e Rousseau, Hassan, Measham, \& Lashley (2008) produziram estudos, respectivamente, na Holanda e no Canadá, cujos achados não evidenciaram maior percepção de queixas por parte de adolescentes imigrantes se comparados a não-imigrantes. A pesquisa de Vollebergh et al. (2005) com jovens não-ocidentais também envolveu dados coletados com seus pais e professores, que, diferentemente, sinalizaram indícios de sofrimento maiores que os relatados pelos adolescentes; e os convocaram a defender a multiplicidade de fontes de informações.

No que se refere à idade à época da imigração, Patterson, Kyu e Georgiades (2013) realizaram investigação que verificou maior risco para alterações no humor e transtornos de ansiedade de crianças que chegaram ao Canadá com menos de 6 anos de idade em comparação com aqueles que imigraram acima dos 18 anos. Tais resultados reforçam a demanda por programas de intervenção e prevenção específicos para a população imigrante infantil.

\section{Propostas de intervenção}

Dentre os trabalhos categorizados neste eixo, foram incluídos os estudos voltados para a mensuração dos impactos psicológicos, na compreensão de que o desenvolvimento de instrumentos tem o propósito científico e assistencial que pode se configurar como interventivo. Suarez-Morales, Dillon e Szapocznik (2007) avaliam as propriedades psicométricas do Inventário de Estresse Aculturativo para a Infância (ASIC - sigla em inglês) em uma amostra de 139 crianças hispânicas. Houve adequação nos quesitos de consistência interna e confiabilidade, além de terem sido obtidas estimativas suficientes da convergente, discriminante e validade preditiva, sendo discutidas as implicações futuras do uso de tal recurso junto a crianças imigrantes. 
Pág 35

Os modelos explicativos da doença, os mecanismos de enfrentamento e o comportamento na busca por ajuda variam em cada cultura, e o entendimento dessas diferenças pode ser significativo na garantia do acesso aos serviços de saúde mental e manutenção da assistência aos imigrantes, com efetiva minimização dos fatores de risco e potencialização dos fatores de proteção do adoecimento (Javier et al., 2014).
Também realizado com jovens hispânicos, o estudo do Inventário de Estresse para adolescentes latino-americanos (HSI-A - sigla em inglês), teve o propósito de desenvolver uma medida culturalmente informada de estresse. Com quesitos voltados para aculturação, educação, família, uso de drogas, vida comunitária, discriminação, o instrumento apresentou coeficiente de confiabilidade esperado (Cervantes, Fisher, Córdova, \& Napper, 2012).

Quanto as possibilidades interventivas ante as crianças imigrantes involuntárias, percebe-se uma sensibilização dos artigos para a atenção às especificidades culturais na atuação prática (Masaud, McNicholas, \& Skokauskas, 2010; Pumariega, Rogers, \& Rothe, 2005; Walker, 2005). Reflete-se que o local de origem daquele que adoece fornece dados sobre as características da saúde da população da região, mas, acima de tudo, as informações culturais poderão traduzir as interpretações, explicações e padrões de enfrentamento daquela população diante da doença (Skokauskas \& Clarke, 2009).

Marks, Ejesi e Coll (2014) sugerem que políticas públicas sejam formuladas de modo a garantir tratamentos culturalmente apropriados, treinamento específico para os profissionais que trabalham com pequenos refugiados, e apoio às crianças para que aprendam a língua e se identifiquem com aspectos cultuais do país de acolhimento, sem perder seus laços com a cultura. Nesse sentido um projeto realizado junto a assistentes sociais prestou serviços às crianças requerentes de refúgio, em que os profissionais passaram a atentar-se as devolutivas das crianças acerca do atendimento recebido e, a partir dessas informações, pretendem se adequar para atendê-las de maneira efetiva (Warwick, Neville, \& Smith, 2006).

Faz-se necessário que os profissionais de saúde estejam atentos à diversidade socioeconômica, étnica e religiosa dos refugiados, a fim de oferecer apoio adequado às especificidades de cada população. Serviços e profissionais de saúde sensíveis à cultura facilitam a expressão de experiências, necessidades e obstáculos vivenciados por grupos minoritários no acesso e recurso à saúde psicológica (Javier et al., 2014; Nadeau \& Measham, 2006). Os modelos explicativos da doença, os mecanismos de enfrentamento e o comportamento na busca por ajuda variam em cada cultura, e o entendimento dessas diferenças pode ser significativo na garantia do acesso aos serviços de saúde mental e manutenção da assistência aos imigrantes, com efetiva minimização dos fatores de risco e potencialização dos fatores de proteção do adoecimento (Javier et al., 2014).

Tendo apresentado os principais resultados desta revisão de literatura acerca da saúde mental de crianças imigrantes, passa-se, então, às considerações finais do trabalho. De modo sintético e com vias avaliativas são feitas reflexões sobre os alcances deste estudo, assim como a respeito de suas limitações, para seguir no incremento de trabalhos que enfoquem a questão do desenvolvimento emocional de crianças imigrantes.

\section{Considerações finais}

O presente estudo se propôs revisar a literatura científica disponibilizada nos últimos treze anos em bases disponíveis eletronicamente acerca dos impactos psicológicos da imigração involuntária na infância. Acredita-se que o caráter original do estudo e a sua relevância atual diante o cenário internacional de crescente fluxo migratório possibilitem a sistematização dos estudos para pesquisas futuras e fomente a discussão sobre a temática. 
Percebe-se que a inexistência de publicações nacionais (Brasil) na modalidade de artigos científicos direcionados aos pequenos imigrantes demanda urgente direcionamento de estudos à área. A ampliação para investigações que abarque livros e revistas não indexadas como dados para coleta, por exemplo, certamente identificará outros achados. Acrescenta-se, ainda, que a busca apenas por artigos disponíveis gratuitamente se evidencia como uma limitação desta pesquisa. Sugere-se que novos estudos possam incluir também artigos acessíveis mediante aquisição financeira, fator que tende a expandir os resultados. Além disso, acredita-se que a utilização de outros descritores e/ou a amplitude nas combinações entre os termos possivelmente estenderá os resultados na pesquisa. Ademais embora o foco da revisão tenha se direcionado para os seguintes tópicos: "Desafios do país de acolhimento", "Impactos psicológicos" e "Propostas de intervenção" deve-se atentar para os temas, tipos de delineamento metodológicos, predileções nas abordagens utilizadas no sentido de questionar os resultados, de problematizá-los e de entender que há um panorama - ser criança, a cultura, a língua, presença ou ausência dos familiares, deixar forçosamente o país de origem - quando se pretende debruçar-se sobre esse assunto.

Considera-se, por fim, que os achados referentes à revisão da literatura acerca dos impactos psicológicos da imigração involuntária na infância apontam para o sofrimento psíquico que as crianças refugiadas vivenciam. Faz-se o alerta, então, à necessidade de produção científica sobre as temáticas retratadas. Sinaliza-se a importância de produzir Políticas Públicas, especialmente na Atenção Básica, que garantam práticas humanizadas ao considerar as singularidades e as diferenças culturais constitutivas dessas crianças, que tão precocemente se deparam com situações fronteiriças a vida.

\section{Referëncias}

Alto Comissariado das Nações Unidas para Refugiados (ACNUR). (2014). Refúgio no Brasil: uma análise estatística (janeiro de 2010 a outubro de 2014). Recuperado de http://www.acnur.org/fileadmin/Documentos/portugues/Estatisticas/Refugio no Brasil 2010 2014.pdf

Antunes, J.A. (2017). Refugiados e saúde mental: acolher, compreender e tratar. Psicologia, Saúde \& Doenças, 18(1), 115-130. Recuperado de http://www.scielo.mec. $\mathrm{pt} /$ scielo.php?script $=$ sci_arttext $\&$ pid $=$ S164500862017000100010

Becker, A. P. S., \& Martins Borges, L. (2015). Dimensões psicossociais da imigração no contexto familiar. Boletim Academia Paulista de Psicologia, 35(88), 126-144. Recuperado de http://pepsic.bvsalud.org/scielo.php?script=sci arttext\&pi$\underline{d}=S 1415711 \times 2015000100009 \& \operatorname{lng}=p t \& t \operatorname{lng}=p t$

Beiser, M., Goodwil, A. M., Albanese, P. McShane, P., \& Nowakowski, M. (2014). Predictors of immigrant children's mental health in Canada: selection, settlement contingencies, culture, or all of the above? Soc Psychiatry Psychiatr Epidemiol., 49(5), 743-756. doi: http://dx.doi-org.ez119.periodicos.capes.gov.br/10.1007/ $\underline{\text { s00127-013-0794-8 }}$

Beiser, M., Hamilton, H., Rummens, J. A., Oxman-Martinez, J., Ogilvie, J., Humphrey, C., \& Armstrong, R. (2010). Predictors of emotional problems and physical aggression among children of Hong Kong Chinese, Mainland Chinese and Filipino immigrants to Canada. Soc Psychiatry Psychiatr Epidemiol., 45(10), 1011-1021. doi: http://dx. doi-org.ez46.periodicos.capes.gov.br/10.1007/s00127-009-0140-3.

Bezerra, C. B. (2016). Distantes do berço: impactos psicológicos da imigração na infância. 2016. Dissertação em Psicologia, Programa de Pós-Graduação em Psicologia, Universidade Federal de Santa Catarina. 
Bronstein, I., Montgomery, P., \& Dobrowolski, S. (2012). PTSD in asylum-seeking male adolescents from Afghanistan. J Trauma Stress., 25(5), 551-557. doi: http://dx. doi-org.ez46.periodicos.capes.gov.br/10.1002/jts. 21740

Buriel, R. (2012). Historical, socio-cultural and conceptual issues to consider when researching Mexican American children and families, and other Latino subgroups. Psychosocial Intervention, 21(3), 291-303. doi: https://dx.doi.org/10.5093/in2012a26a26

Calzada, E. J., Huang, K. Y., Linares-Torres, H., Singh, S. D., \& Brotman, L. (2014). Maternal Familism and early childhood functioning in Mexican and Dominican immigrant families. J Lat Psychol., 2(3), 156-171. doi: http://dx.doi-org.ez46.periodicos. capes.gov.br/10.1037/lat0000021

Castro, A., Bermúdez, M. P., \& Buela-Casal, G. (2009). Variables relacionadas con la infección por VIH en adolescentes inmigrantes latinoamericanos: una revisión revista argentina de clínica psicológica. Revista Argentina de Clínica Psicológica, 3, 227-240. Recuperado de AcademicOneFile, http://link.galegroup.com/apps/ doc $/ A 254972305 / A O N E ? u=c a p e s \&$ sid=AONE\&xid=73a0e9b1

Cervantes, R. C., Cardoso, J. B., \& Goldbach, J. T. (2015). Examining differences in culturally based stress among clinical and nonclinical Hispanic adolescents. Cultur Divers Ethnic Minor Psychol., 21(3), 458-467. doi: http://dx.doi-org.ez46.periodicos.capes.gov.br/10.1037/a0037879

Cervantes, R. C., Fisher, D. G., Córdova, D., \& Napper, L. E. (2012). The Hispanic stress inventory-adolescent version: a culturally informed psychosocial assessment. Psychol Assess, 24(1), 187-196. doi. http://dx.doi-org.ez46.periodicos.capes.gov. br/10.1037/a0025280

Chuang, S. S., \& Gielen, U. P. (2009). Understanding immigrant families from around the world: introduction to the special issue. J Fam Psychol, 23(3), 275-278. doi: http://dx.doi-org.ez46.periodicos.capes.gov.br/10.1037/a0016016.

Comitê Nacional para os Refugiados (CONARE). (2017). Refúgio em Números. Ministério da Justiça e Segurança Pública. Secretaria Nacional de Justiça.

Cook, L. B., Brown, J. D., Loder, S., \& Wissow, L. (2014). Acculturation differences in communicating information about child mental health between Latino parents and primary care providers. J Immigr Minor Health, 16(6), 1093-102. doi: http:// dx.doi-org.ez46.periodicos.capes.gov.br/10.1007/s10903-014-0010-2

Ellis, B. H., MacDonald, H. Z., Lincoln, A. K., \& Cabral, H. J. (2008). Mental health of Somali adolescent refugees: the role of trauma, stress and perceived discrimination. Journal of Consulting and Clinical Psychology, 76(2), 184-193. doi: http:// dx.doi-org.ez46.periodicos.capes.gov.br/10.1037/0022-006X.76.2.184

Erol, N., Simsek, Z., Öner, O., \& Munir, K. (2005). Effects of internal displacement and resettlement on the mental health of Turkish children and adolescents. Eur Psychiatry, 20(2), 152-157. doi: http://dx.doi-org.ez46.periodicos.capes.gov. br/10.1016/j.eurpsy.2004.09.033

Flink, I. J., Restrepo, M. H., Blanco, D. P., Ortegon, M. M., Enriquez, C. L., Beirens, T., \& M., Raat, H. (2013). Mental health of internally displaced preschool children: a cross-sectional study conducted in Bogotá, Colombia. Soc Psychiatry Psychiatr Epidemiol, 48(6), 917-926. doi: http://dx.doi-org.ez46.periodicos. capes.gov.br/10.1007/s00127-012-0611-9

Frankenberg, E., Kupper, K., Wagner, R., \& Bongard, S. (2013). Immigrant youth in germany psychological and sociocultural adaptation. European Psychologist, 18(3), 158-168. doi: http://dx.doi-org.10.1027/1016-9040/a000154

Georgiades, K., Boyle, M. H., \& Duku, E. (2007). Contextual influences on children's mental health and school performance: the moderating effects of family immi- 
grant status. Child Dev., 78(5), 1572-191. doi: http://dx.doi-org.ez46.periodicos. capes.gov.br/10.1111/j.14678624.2007.01084.x

González, A. M., Cuxart, M. P., \& Peco, S. G. (2012). Actuaciones educativas para la convivencia en centros con alumnado inmigrante. Revista Interuniversitaria de Formación del Profesorado, 26(1), 133-144. Recuperado de http://www.aufop. com/aufop/uploaded files/revistas/13528246646.pdf\#page=133

Gupta, T., Rogers-Sirin, T., Okazaki, S., Ryce, P., \& Sirin, S. R. (2014). The role of collective self-esteem on anxious-depressed symptoms for Asian and Latino children of immigrants. Cultur Divers Ethnic Minor Psychol., 20(2), 220-230. doi: http:// dx.doi-org.ez46.periodicos.capes.gov.br/10.1037/a0035022

Hadfield, K., Ostrowski, A., \& Ungar, M. (2017). What can we expect of the mental health and well-being of Syrian refugee children and adolescents in Canada? Canadian Psychology/Psychologie canadienne, 58(2), 194-201. doi: http://dx.doi-org. ez46.periodicos.capes.gov.br/10.1037/cap0000102

Javier, J. R., Supan, J., Lansang, A., Beyer, W., Kubicek, K., \& Palinkas, L. A. (2014). Preventing filipino mental health disparities: perspectives from adolescents, caregivers, providers, and advocates. Asian Am J Psychol., 5(4), 316-324. doi: http:// dx.doi-org.ez46.periodicos.capes.gov.br/10.1037/a0036479

Katsiaficas, D., Suárez-Orozco, C., Sirin, S. R., \& Gupta, T. (2013). Mediators of the relationship between acculturative stress and internalization symptoms for immigrant origin youth. Cultur Divers Ethnic Minor Psychol., 19(1), 27-37. doi: http://dx. doi-org.ez46.periodicos.capes.gov.br/10.1037/a0031094

Kostelny, K., \& Ondoro, K. (2016). Structural violence and the everyday stresses of internally displaced children in Somalilandand Puntland. Peace and Conflict: Journal of Peace Psychology, 22(3), 226-235. doi: http://dx.doi-org.ez46.periodicos. capes.gov.br/10.1037/pac0000171

Kronick, R., Rousseau, C., \& Cleveland, J. (2015). Asylum-seeking children's experiences of detention in Canada: a qualitative study. Am J Orthopsychiatry, 85(3), 287-294. doi: http://dx.doi-org.ez46.periodicos.capes.gov.br/10.1037/ ort0000061

Lauritzen, C., \& Sivertsen, H. (2012). Children and families seeking asylum in northern norway: Living conditions and mental health. International Migration, 60(6), 195-210. doi: http://doi-org.ez119.periodicos.capes.gov.br/10.1111/ j.1468-2435.2012.00774.x

Leiner, M. A., Straus, D. C. Balcazar, H., \& Handal, G. (2006). Screening Mexicans for psychosocial and behavioral problems during pediatric consultation. Revista de Investigacion Clinica, 59(2), 116-123. Recuperado de http://web-b-ebscohost.ez119.periodicos.capes.gov.br/ehost/pdfviewer/pdfviewer?vid=2\&sid=449fa21d-b0a5-4d8f-be2c-d6d3b5686d13\%40sessionmgr103

Lien, L., Haavet, O. R., Thoresen, M., Heyerdahl. S., \& Bjertness, E. (2007). Mental health problems, negative life events, perceived pressure and the frequency of acute infections among adolescents. Results from a cross-sectional, multicultural, population-based study. Acta Paediatr, 96(2), 301-306. doi: http://dx.doi-org. ez46.periodicos.capes.gov.br/10.1111/j.1651-2227.2007.00084.x

Lipsicas, B. C., \& Mäkinen, H. I. (2010). Immigration and suicidality in the young. Can J Psychiatry, 55(5), 274-281. Recuperado de http://link.galegroup.com/apps/doc/ A228545578/AONE?u=capes\&sid=AONE\&xid=f9315479

López-Pozos, C. (2009). El costo emocional de la separación en niños migrantes: un estudio de caso de migración familiar entre Tlaxcala y California. Agricultura, Sociedad y Desarrollo, 6(1), 81-103. Recuperado de http://www.scielo.org.mx/scielo.php?script=sci arttext\&pid=S187054722009000100004\&lng=en\&tlng=es 
Loreka, A., Ehntholt, K., Nesbitt, A., Wey, E., Githinji, C., Rossor, E., \& Wickramasinghe, R. (2009). The mental and physical health difficulties of children held within a British immigration detention center: a pilot study. Child Abuse Negl, 33(9), 573-85. doi: http://dx.doi-org.ez46.periodicos.capes.gov.br/10.1016/j.chiabu.2008.10.005

Mace, A. O., Mulheron, S., Jones, C., \& Cherian, S. (2014). Educational, developmental and psychological outcomes of resettled refugee children in Western Australia: A review of school of special educational needs: Medical and mental health input. J Paediatr Child Health, 50(12), 985-92. doi: http://doi-org.ez119.periodicos. capes.gov.br/10.1111/jpc. 12674

Marks, A. K., Ejesi, K., \& Coll, C. G. (2014). Understanding the U.S: immigrant paradox in childhood and adolescence. Child Development Perspectives, 8(2), 59-64. doi: http://dx.doi-org.ez46.periodicos.capes.gov.br/10.1111/cdep.12071

Martins-Borges, L. (2017). Migrações involuntárias e impactos psíquicos: a mediação da cultura. In Peres, R. S., Hashimoto, F., Casadore, M. M., \& Braz, M. V. (Org.). Sujeito contemporâneo. (pp. 169-186). São Carlos: Edufscar.

Masaud, T., McNicholas, F., \& Skokauskas, N. (2010). Overcoming the challenges of managing mental health in migrant children. Pediatric Health, 4(6), 603-611. doi: http://dx.doi-org.ez119.periodicos.capes.gov.br/10.2217/phe.10.69

Moleiro, C., \& Gonçalves, M. (2010). Saúde na diversidade: desenvolvimento de serviços de saúde mental sensíveis à cultura. Análise Psicológica, 3(28), 505-515. doi: https://doi.org/10.14417/ap.353

Nadeau, L., \& Measham, T. (2006). Caring for migrant and refugee children: challenges associated with mental health care in pediatrics. J Dev Behav Pediatr., 27(2), 145154. doi: http://dx.doi-org.ez46.periodicos.capes.gov.br/10.1097/00004703200604000-00013

Nielsen, S. S., Norredam, M., Christiansen, K. L., Obel, C., Hilden, J., \& Krasnik, A. (2008). Mental health among children seeking asylum in Denmark - the effect of length of stay and number of relocations: a cross-sectional study. BMC Public Health, 8(293), 1-9. doi: https://doi.org/10.1186/1471-2458-8-293

Patterson, B., Kyu, H. H., \& Georgiades, K. (2013). Age at immigration to Canada and the occurrence of mood, anxiety and substance use disorders. Can J Psychiatry., 58(4), 210-217. doi: https://doi.org/10.1177/070674371305800406

Perdigão, D. M., Herlinger, M., \& White, O. M. (Orgs.). (2012). Teoria e prática da pesquisa aplicada. Rio de Janeiro, RJ: Elsevier.

Pfeifer, J. H., Rubble, D. N., Bachman, M. A., Alvarez, J. M., Cameron, J. A., \& Fuligni, A. J. (2007). Social identities and intergroup bias in immigrant and nonimmigrant children. Dev Psychol., 43(2), 496-507. doi: https://dx.doi-org.ez46.periodicos. capes.gov.br/10.1037/0012-1649.43.2.496

Pumariega, A. J., Rogers, K., \& Rothe, E. (2005). Culturally competent systems of care for children's mental health: advances and challenges. Community Ment Health J., 47(5), 539-555. doi: http://dx.doi-org.ez46.periodicos.capes.gov.br/10.1007/ s10597-005-6360-4

Pumariega, A. J., \& Rothe, E. (2010). Leaving no children or families outside: the challenges of immigration. Am J Orthopsychiatry, 80(4), 505-15. doi: https:// doi-org.ez119.periodicos.capes.gov.br/10.1111/j.1939-0025.2010.01053.x

Rousseau, C., Hassan, G., Measham, T., \& Lashley, M. (2008). Prevalence and correlates of conduct disorder and problem behavior in Caribbean and Filipino immigrant adolescents. Eur Child Adolesc Psychiatry.,17(5), 264-273. doi: https://dx.doi-org. ez46.periodicos.capes.gov.br/10.1007/s00787-007-0640-1

Skokauskas, N., \& Clarke, D. (2009). Mental health of immigrant children: A new challenge for child and adolescent psychiatry services in Ireland. Child Care 
in Practice, 15(3), 227-233. doi: http://dx.doi-org.ez46.periodicos.capes.gov. br/10.1080/13575270902891081

Spiegler, O., Verkuyten, M., Thijs, J., \& Leyendecker, B. (2016). Low ethnic identity exploration undermines positive interethnicrelations: A study among Turkish immigrant-origin youth. Cultural Diversity and Ethnic Minority Psychology, 22(4), 495-503. doi: http://dx.doi-org.ez46.periodicos.capes.gov.br/10.1037/cdp0000090

Suarez-Morales, L., Dillon, F. R., \& Szapocznik, J. (2007). Validation of the acculturative stress inventory for children. Cultur Divers Ethnic Minor Psychol., 13(3), $216-$ 224. doi: http://dx.doi.org/10.1037/1099-9809.13.3.216

Trickett, E. J \& Jones, C. J. (2007). Adolescent culture brokering and family functioning: a study of families from Vietnam. Cultur Divers Ethnic Minor Psychol, 13(2), 143-150. doi: https://dx.doi-org.ez46.periodicos.capes.gov.br/10.1037/1099-9809.13.2.143

Tummala-Narra, P. (2014). Cultural Identitiy in the context of trauma and immigration from a psychoanalytic perspective. Psychoanalytic Psychology, 31(3), 396-409. doi: http://dx.doi.org/10.1037/a0036539

United Nations High Commissioner for Refugees (UNHCR). (2017). Global trends: Forced displacement in 2016. doi: https://www.unhcr.org/statistics/unhcrstats/5943e8a34/global-trends-forced-displacement-2016.html

Vollebergh, W. A, Have, M., Dekovic, M. Oosterwegel, A., Pels, T., Veenstra, R., Verhulst, F. (2005). Mental health in immigrant children in the Netherlands. Soc Psychiatry Psychiatr Epidemiol, 40(6), 489-496. doi: http://dx.doi-org.ez119.periodicos.capes. gov.br/10.1007/s00127-005-0906-1

Walker, S. (2005). Towards culturally competent practice in child and adolescent mental health. International Social Work, 48(1), 49-62. doi:http://dx.doi-org.ez46.periodicos.capes.gov.br/10.1177/0020872805048708

Warwick, l., Neville, R., \& Smith, K. (2006). My life in Huddersfield: supporting young asylum seekers and refugees to record their experiences of living in huddersfield. Social Work Education, 25(2), 129-137. doi: https://dx.doi-org.ez46.periodicos.capes.gov.br/10.1080/02615470500487309

Ziol-Guest, K. M., \& Kalil, A. (2012). Health and medical care among the children of immigrants. Child Dev., 83(5), 1494-500. doi: http://doi-org.ez119.periodicos.capes.gov.br/10.1111/j.1467-8624.2012.01795.x 